\title{
THE CLOSURE OF THE SIMILARITY ORBIT OF A HILBERT SPACE OPERATOR
}

\author{
BY CONSTANTIN APOSTOL, DOMINGO A. HERRERO AND DAN VOICULESCU
}

1. Introduction. Let $H$ be a complex separable infinite-dimensional Hilbert space and let $L(H)$ be the algebra of all (bounded linear) operators acting on $H$. The similarity orbit of $T \in L(H)$ is the subset

$$
S(T)=\left\{W T W^{-1}: W \in L(H) \text { is invertible }\right\} .
$$

The purpose of this note is to announce the "almost complete" solution of the problem of characterizing the (norm) closure $S(T)^{-}$of $S(T)$ in simple terms. Our results reduce the whole problem to the analysis of a very peculiar class of nilpotent operators and their compact perturbations. Complete results will appear elsewhere.

2. The main result. Assume that $A \in S(T)^{-}$, i.e., $\left\|A-W_{n} T W_{n}^{-1}\right\| \rightarrow 0$ $(n \rightarrow \infty)$ for a suitable sequence $\left\{W_{n}\right\}_{n=1}^{\infty}$ of invertible operators. Since the spectrum of every operator in the sequence $\left\{W_{n} T W_{n}^{-1}\right\}_{n=1}^{\infty}$ coincides with the spectrum $\sigma(T)$ of $T$ and, moreover, every single piece of $\sigma\left(W_{n} T W_{n}^{-1}\right)$ (essential spectrum, left or right essential spectrum, normal eigenvalues, etc.) coincides with the corresponding piece of $\sigma(T)$, it is not difficult to see, by using the upper semicontinuity of separate parts of the spectrum (see, e.g., [5, Theorem 3.16]) that $A$ necessarily satisfies

(0) $\sigma(A) \supset \sigma(T)$ and each component of $\sigma(A)$ intersects $\sigma(T)$.

Furthermore, if $f$ is an analytic function defined on a neighborhood of $\sigma(A)$ and we define $f(A)$ via Riesz-Dunford functional calculus, then it is easily seen that $\left\|f(A)-f\left(W_{n} T W_{n}^{-1}\right)\right\| \longrightarrow 0(n \rightarrow \infty)$. If $\sigma$ is a clopen subset of $\sigma(A)$, $f(\lambda) \equiv 1$ on a neighborhood of $\sigma$ and $f(\lambda) \equiv 0$ on a neighborhood of $\sigma(A) \backslash \sigma$, then $P(\sigma ; A)=f(A)$ is the Riesz' idempotent corresponding to $\sigma[6$, Chapter $\mathrm{XIV]}$. Recall that $\lambda \in \sigma(A)$ is a normal eigenvalue if $\lambda$ is an isolated point of $\sigma(A)$ and $\lambda \notin \sigma_{e}(A)$; equivalently: $\lambda$ is an isolated point of $\sigma(A)$ and $P(\{\lambda\} ; A)$ is a finite rank operator. (The set of all normal eigenvalues of $A$ will be denoted by $\sigma_{0}(A)$.)

The continuity properties of the functional calculus imply that

(i) If $\lambda \in \sigma_{0}(A)$, then $\operatorname{rank} P(\{\lambda\} ; A)=\operatorname{rank} P(\{\lambda\} ; T)$.

Received by the editors May 4, 1981.

1980 Mathematics Subject Classification. Primary 47A66, 47A99; Secondary 47A53, $47 \mathrm{A5} 5$.

C일 1982 American Mathematical Society 0273-0979/81/0000-0083/\$02.50 
Let $K(H)$ denote the ideal of all compact operators and let $\pi: L(H) \rightarrow$ $L(H) / K(H)$ be the canonical projection of $L(H)$ onto the quotient Calkin algebra; $\pi(T)$ will also be denoted by $\widetilde{T}$. Let $\sigma_{e}(T)=\sigma(\widetilde{T})$ be the essential spectrum of $T$, let $\sigma_{l e}(T)\left(\sigma_{r e}(T)\right)$ denote the left (right, resp.) essential spectrum of $T$ and let $\rho_{s-F}(T)$ (the complement of $\sigma_{l e}(T) \cap \sigma_{r e}(T)$ in the complex plane $\mathbf{C}$ ) be the semi-Fredholm domain of $T$ (the reader is referred to [5] for definition and properties of semi-Fredholm operators). The well-known stability properties of the semi-Fredholm operators and the upper semicontinuity of separate parts of the essential spectrum imply that

(iii) $\sigma_{e}(A) \supset \sigma_{e}(T)$ and each component of $\sigma_{e}(A)$ intersects $\sigma_{e}(T)$ and

(iv) $\rho_{s-F}(A) \subset \rho_{s-F}(T)$ and $\operatorname{ind}(\lambda-A)=\operatorname{ind}(\lambda-T)$ for all $\lambda \in \rho_{s-F}(A)$.

Furthermore, if (following [1]) we define

$$
\min . \operatorname{ind}(\lambda-T)=\min \{\operatorname{nul}(\lambda-T), \operatorname{nul}(\lambda-T) *\}, \lambda \in \rho_{s-F}(T),
$$

where nul $B$ is the (topological) dimension of the kernel, $\operatorname{ker} B$, of $B$ and $B^{*}$ is the adjoint of $B$ in $L(H)$, then

(ii) If $\lambda \in \rho_{s-F}(A)$, then min. $\operatorname{ind}(\lambda-A)^{k} \geqslant \min$. ind $(\lambda-T)^{k}$ for all $k=$ $1,2,3, \ldots$ (Observe that (i)-(iv) imply (0).)

It is easily seen that if $A \in S(T)^{-}$, then $\widetilde{A} \in S(\widetilde{T})^{-}$(where similarity is defined in terms of the invertible elements of the Calkin algebra). The continuity properties of the functional calculus in the Calkin algebra imply that

(v) If $f$ is an analytic function defined on a neighborhood of $\sigma_{e}(A)$ such that $f(\widetilde{T})=0$, then $f(\widetilde{A})=0$.

Let $\rho: L(H) / K(H) \rightarrow L\left(H_{\rho}\right)$ be a faithful unital *-representation. If $\lambda$ is an isolated point of $\sigma_{e}(T)$, then $\rho(\widetilde{T}) \sim\left(\lambda+Q_{\lambda}\right) \oplus R_{\lambda}$, where $Q_{\lambda}$ is a quasinilpotent operator and $\lambda \notin \sigma\left(R_{\lambda}\right)$. For every $\lambda \in \mathbf{C}$ define

$$
k(\lambda ; \widetilde{T})= \begin{cases}0 & \text { if } \lambda \notin \sigma_{e}(T), \\ n & \text { if } Q_{\lambda} \text { is a nilpotent of order } n \\ \infty & \text { otherwise. }\end{cases}
$$

With this notation in mind, it is not difficult to deduce from (iii) and (v) that

(00) If $\sigma$ is a component of $\sigma_{e}(A)$ and $\sigma$ is not a singleton, then $\sigma \cap$ $\left\{\lambda \in \sigma_{e}(T): k(\lambda ; \tilde{T})=\infty\right\} \neq \varnothing$.

THEOREM 1 (RESTRICTED FORM). If $T \in L(H)$ and $k(\lambda ; \widetilde{T})=\infty$ for all $\lambda \in \sigma_{e}(T)$, then $S(T)^{-}=\{A \in L(H): A$ satisfies (i)-(iv) $\}$.

\section{Pathological behavior associated with nilpotent elements of the Calkin}

algebra. It is clear from (iii), (v) and (00) that if $\lambda$ is an isolated point of $\sigma_{e}(A)$ 
and $k(\lambda ; \widetilde{A})=n$, then $\lambda$ is an isolated point of $\sigma_{e}(T)$ and $n \leqslant k(\lambda ; \widetilde{T}) \leqslant \infty$. Unfortunately, this necessary condition (together with (i)-(v)) is not sufficient to guarantee that $A \in S(T)^{-}$. The reason is the peculiar behavior of certain nilpotent elements of $L(H) / K(H)$. To make it more precise, we shall need the following definition:

If $\lambda$ is an isolated point of $\sigma_{e}(T)$ such that $k(\lambda ; \widetilde{T})=n$ for some $n, 2 \leqslant$ $n<\infty$, and $Q_{\lambda}$ is similar to a Jordan cell of order $n$, we shall say that $\lambda$ is a megambic ${ }^{1}$ point of $T$ (affiliated with $q_{k(\lambda ; \tilde{T})}^{(\infty)}$ ).

Then we have

(vi) If $\lambda$ is a megambic point for $A$ and $k(\lambda ; \widetilde{T})=k(\lambda ; \widetilde{A})$, then $\lambda$ is a megambic point for $T$; and

(vii) If, moreover, $\lambda$ belongs to the boundary $\partial \sigma(A)$ of $\sigma(A)$ and $\sigma$ is a clopen subset of $\sigma(A)$ such that $\sigma \cap \sigma_{e}(A)=\{\lambda\}$ then $\sigma$ is a clopen subset of $\sigma(T)$ and $A \mid \operatorname{ran} P(\sigma ; A)$ is unitarily equivalent to an operator in $S(T \mid \operatorname{ran} P(\sigma ; T))^{-}$.

Theorem 1 (General form). Let $T \in L(H)$; then $S(T)^{-}=\{A \in L(H)$ : A satisfies (i)-(vii)\}.

The results contained in the preprint [3] by J. Barria and D. A. Herrero about closure of similarity orbits of nilpotent operators are essentially correct but, unfortunately, the proofs given there contain several errors. Correct proofs of the same results (indeed, slightly better ones) for the separable case (which is the only one considered here) has been almost simultaneously obtained by C. Apostol and D. Voiculescu in [2], by using a different approach. The notion involved in the definition of megambic point has been taken from this last reference.

Theorem 1 reduces the general problem of characterizing $S(T)^{-}$for an arbitrary $T$ in $L(H)$ to that of characterizing

$$
\begin{aligned}
S^{+}\left(0^{(j)} \oplus q_{n}^{(\infty)}+K\right)= & \left\{A \in S\left(0^{(j)} \oplus q_{n}^{(\infty)}+K\right)^{-}: A\right. \text { is unitarily } \\
& \text { equivalent to } \left.0^{(j)} \oplus q_{n}^{(\infty)}+C\right\},
\end{aligned}
$$

where $n \geqslant 2,0 \leqslant j<n, 0^{(j)}$ denotes the zero operator acting on $\mathbf{C}^{j}, q_{n}$ is the Jordan nilpotent cell of order $n$, and $K, C$ are compact operators. This promises to be a very difficult problem. Partial results will be included in the publication of the complete results.

In fact, the answer to the above problem is known for several particular compact perturbations of $0^{(j)} \oplus q_{k}^{(\infty)}$. Moreover, given $T$ in $L(H)$ and $\epsilon>0$, there exists $K_{\epsilon} \in K(H)$ such that $\left\|K_{\epsilon}\right\|<\epsilon$ and the closure of $S\left(T+K_{\epsilon}\right)$ admits a complete characterization in simple terms (namely, $K_{\epsilon}$ can be chosen so that the condition (vii) can be replaced by the more simple statement Calderón.

${ }^{1}$ This expression has been suggested to the second author by Professor Alberto P. 
(vii') If $\lambda$ and $\sigma$ satisfy the conditions of (vi) and $T \mid \operatorname{ran} P(\sigma ; T)$ is similar to a compact perturbation of $0^{(j)} \oplus q_{n}^{(\infty)}$, then $A \mid \operatorname{ran} P(\sigma ; A)$ is also similar to a compact perturbation of $0^{(j)} \oplus q_{n}^{(\infty)}$ (provided $T$ has been replaced by $T+K_{\epsilon}$ )).

4. The closure of the similarity orbit in the Calkin algebra. As a corollary of our previous observations, we obtain a complete characterization of the norm closure of an element of the Calkin algebra. The set of all megambic points of $T$ will be denoted by $\sigma_{m e}(T)$.

THEOREM 2. Let $\widetilde{T} \in L(H) / K(H)$; then

$$
S(\widetilde{T})^{-}=\{\tilde{A} \in L(H) / K(H): A \text { satisfies (iii)-(vi) }\} \text {. }
$$

5. Consequences. Many (very general) results on closure of classes of operators defined in terms of different parts of the spectrum can be easily derived from the previous theorems. Namely, we have the following

Corollary 3. Let $X, \Omega$ and $\Lambda$ be three pairwise disjoint subsets of $\mathrm{C}$ such that $X$ is compact, $\Omega$ is open and bounded, $X$ contains the boundary $\partial \Omega$ of $\Omega$ and $X \cup \Omega \cup \Lambda \neq \varnothing$. Let $\left\{\Omega_{j}\right\}$ be an enumeration of the components of $\Omega$ and let $X_{0}=\left\{\mu_{r}\right\}$ be a subset of isolated points of $X$. If $R$ is the family of all those operators $T$ in $L(H)$ satisfying the conditions

(a) $X \cup \Omega \subset \sigma(T) \subset X \cup \Omega \cup \Lambda$;

(b) $\lambda-T$ is a Fredholm operator of $\operatorname{ind}(\lambda-T)=m_{j}$ and $\min . \operatorname{ind}(\lambda-T)$ $=n_{j} \geqslant 0$ for all $\lambda \in \Omega_{j}, j=1,2, \ldots$;

(c) $\mu_{r}$ is a megambic point of $T$ affiliated with $q_{k_{r}}^{(\infty)}, r=1,2, \ldots$; then $R^{-}$is the set of all those $A$ in $L(H)$ such that

(a1) $\sigma(A) \supset X \cup \Omega$, every component of $\sigma(A)$ intersects $X \cup \Omega \cup\left(\Lambda^{-}\right)$ and every component of $\sigma_{l e}(A) \cap \sigma_{r e}(A)$ intersects $X \cup \Lambda^{-}$;

(a2) If $\lambda-A$ is semi-Fredholm and $\operatorname{ind}(\lambda-A) \neq 0$, then $\lambda$ belongs to $\Omega^{-} \cup($ interior $X \cup \Lambda)$;

(a3) If $\lambda-A$ is a Fredholm operator of index zero and

$$
\lambda \in \partial X \backslash\left[\Omega^{-} \cup(\text { interior } \Lambda)\right],
$$

then $\lambda$ is an isolated point of $X$;

(b1) If $\lambda \in \Omega_{j}$ and $\lambda-A$ is semi-Fredholm, then $\operatorname{ind}(\lambda-A)=m_{j}$ and $\min \cdot \operatorname{ind}(\lambda-A) \geqslant n_{j}$;

(c1) If $\mu_{r}$ is an isolated point of $\sigma_{e}(A)$, then $f_{k_{r}}\left(\tilde{A} ; \mu_{r}, A\right)=0$, where

$$
f_{k_{r}}\left(\lambda ; \mu_{r}, A\right)= \begin{cases}\left(\lambda-\mu_{r}\right)^{k_{r}} & \text { on a neighborhood of } \mu_{r}, \\ 0 & \text { on a neighborhood of } \sigma_{e}(A) \backslash\left\{\mu_{r}\right\}\end{cases}
$$


(c2) If $\sigma$ is a component of $\sigma_{e}(A)$ and $\sigma$ is not a singleton, then $\sigma \cap$ $(X \cup \Lambda)$ cannot be a finite subset of $X_{0}$.

Furthermore, if $\Lambda=\varnothing$, then

$$
\mathrm{R}^{-}=\bigcup\left\{S(T)^{-}: T \text { satisfies (a), (b), (c) }\right\} .
$$

Conditions (a), (b), and (c) can be replaced by an arbitrary list of compatible conditions (related with suitable subsets of the spectrum). The answer is always the "obvious" one: The necessary conditions derived from (i)-(vii) are also sufficient.

Analogous results hold for subsets of the Calkin algebra.

6. The distance to a similarity orbit. Recent results of D. A. Herrero [4] on construction of approximants to certain similarity invariant sets of operators provide a partial answer to the problem of estimating the distance to a similarity orbit. Given $T \in L(H)$, let $m_{e}(T)$ denote the essential minimum modulus of $T$, defined by $m_{e}(T)=\min \left\{\lambda \in \sigma\left(\left(\widetilde{T}^{*} \widetilde{T}\right)^{1 / 2}\right)\right\}$.

Proposition 4. If $A, T \in L(H)$ and $k(\lambda ; \tilde{T})=\infty$ for all $\lambda \in \sigma_{e}(T)$, then

$$
\operatorname{dist}[\tilde{A}, S(\tilde{T})]=\operatorname{dist}[A, S(T)+K(H)]=\max \left\{\alpha, \alpha^{*}, \beta\right\} \text {, }
$$

where

$$
\begin{gathered}
\alpha=\sup \left\{m_{e}(\lambda-A): \lambda \in \rho_{s-F}(A) \text { and either } \lambda \notin \rho_{s-F}(T),\right. \\
\text { or } \left.\lambda \in \rho_{s-F}(T) \text { and } \operatorname{ind}(\lambda-T) \neq \operatorname{ind}(\lambda-A)\right\}, \\
\alpha^{*}=\sup \left\{m_{e}(\lambda-A)^{*}: \lambda \in \rho_{s-F}(A) \text { and either } \lambda \notin \rho_{s-F}(T),\right. \\
\text { or } \left.\lambda \in \rho_{s-F}(T) \text { and } \operatorname{ind}(\lambda-T) \neq \operatorname{ind}(\lambda-A)\right\} \\
\Delta_{\gamma}(A)=\left\{\lambda \in \mathbf{C}: \max \left[m_{e}(\lambda-A), m_{e}(\lambda-A)^{*}\right] \leqslant \gamma\right\}
\end{gathered}
$$

and

$$
\beta=\min \left\{\gamma \geqslant 0: \text { Each component of } \Delta_{\gamma}(A) \text { intersects } \sigma_{e}(T)\right\} \text {. }
$$

Furthermore, there exists $L \in S(T)^{-}+K(H)$ such that

$$
\|A-L\|=\operatorname{dist}[A, S(T)+K(H)] .
$$

\section{REFERENCES}

1. C. Apostol, Correction by compact perturbations of the singular behavior of operators, Rev. Roumaine Math. Pures Appl. 21 (1976), 155-175.

2. C. Apostol and D. Voiculescu, Closure of similarity orbits of nilpotent operators (preprint, not for publication), 1977.

3. J. Barría and D. A. Herrero, Closure of similarity orbits of nilpotent operators. II (preprint, not for publication), 1977. 

1981.

4. D. A. Herrero, The distance to a similarity-invariant set of operators (preprint),

5. T. Kato, Perturbation theory for linear operators, Springer-Verlag, New York, 1966.

6. F. Riesz and B. Sz.-Nagy, Functional analysis, Ungar, New York, 1955.

DEPARTMENT OF MATHEMATICS, INSTITUTE FOR SCIENTIFIC AND TECHNICAL CREATION, BD. PACII 220, 77538, BUCHAREST, ROMANIA

DEPARTMENT OF MATHEMATICS, UNIVERSITY OF GEORGIA, ATHENS, GEORGIA 30602

Current address (Domingo A. Herrero): Department of Mathematics, Arizona State University, Tempe, Arizona 85287. 